\title{
Coronary Sinus
}

National Cancer Institute

\section{Source}

National Cancer Institute. Coronary Sinus. NCI Thesaurus. Code C32378.

The coronary vein that terminates in the right atrium and transports deoxygenated blood from the coronary circulation. 